\title{
Synthesis and biological evaluation of some novel 2-phenyl benzimidazole-1-acetamide derivatives as potential anthelmintic agents
}

\author{
RAMESH SAWANT* \\ DEEPALI KAWADE \\ Department of Pharmaceutical \\ Chemistry and PG Studies \\ PDVVPF's College of Pharmacy \\ Post-MIDC, Vilad Ghat \\ Ahmednagar-414111, India
}

\begin{abstract}
The present study describes synthesis of a series of 2-phenyl benzimidazole-1-acetamide derivatives and their evaluation for anthelmintic activity using Indian adult earthworms, Pheretima posthuma. The structure of the title compounds was elucidated by elemental analysis and spectral data. The compounds $4-(\{[2-(4-n i t r o p h e n y l)-1 H-$ -benzimidazol-1-yl]acetyl\}amino) benzoic acid (3a), N-ethyl-2-[2-(4-nitrophenyl)-1H-benzimidazol-1-yl] acetamide (3c), $N$-benzyl-2-[2-(4-nitrophenyl)-1H-benzimidazol-1-yl] acetamide (3d), N-(4-hydroxyphenyl)-2-[2-(4-nitrophenyl)-1H-benzimidazol-1-yl] acetamide (3f), 2-[2-(4-nitrophenyl)-1H-benzimidazol-1-yl]- $N$-phenyl acetamide (3h), 2-[2-(4-chlorophenyl)-1H-benzimidazol-1-yl]- $N$ '-phenylacetohydrazide (3k), 2-[2-(4-chlorophenyl)-1H-benzimidazol-1-yl]-N-(4-nitrophenyl) acetamide (3n) and 2-[2-(4-chlorophenyl)-1H-benzimidazol-1-yl]-N-phenyl acetamide (3q) were found better to paralyze worms whereas $N$-ethyl-2-[2-(4-nitrophenyl)-1H-benzimidazol-1-yl] acetamide (3c), N-(4-nitrophenyl)-2-[2-(4-nitrophenyl)-1H-benzimidazol-1-yl] acetamide (3e), 4-(\{[2-(4-chlorophenyl)-1H-benzimidazol-1-yl]acetyl \}amino) benzoic acid (3j), 2-[2-(4-chlorophenyl)-1H-benzimidazol-1-yl]- $N$-ethyl acetamide (31) and 2-[2-(4-chlorophenyl)-1H-benzimidazol-1-yl]- $N$-phenyl acetamide $(3 q)$ were better to cause death of worms compared to the anthelmintic drug albendazole.
\end{abstract}

Keywords: benzimidazole, anthelmintic activity, Pheretima posthuma

Benzimidazoles are regarded as a promising class of bioactive heterocyclic compounds that exhibit a wide range of biological activities. Their nucleus is a constituent of vitamin B12 and is present in numerous compounds possessing antioxidant $(1,2)$, antiparasitic (3), anthelmintic (4), antiproliferative (5), anti-HIV (6), anti-allergy (7), antiinflammatory (8), antihypertensive (9), antineoplastic (10) and antiulcer activity (11).

* Correspondence; e-mail: sawantrl@yahoo.com 
R. Sawant and D. Kawade: Synthesis and biological evaluation of some novel 2-phenyl benzimidazole-1-acetamide derivatives as potential anthelmintic agents, Acta Pharm. 61 (2011) 353-361.

The literature has revealed that the substitutions at the 1, 2 and 5 positions of the benzimidazole moiety are crucial for exhibiting a wide range of pharmacological activities. Specifically, 2-substituted analogs of benzimidazole are known to be potent biologically active compounds.

2-Arylbenzimidazoles are usually synthesized by classical cyclocondensation of $o$-phenylenediamines with the corresponding carboxylic acids under harsh dehydrating reaction conditions (12) or with aldehydes under oxidative conditions (13). The condensation of $o$-phenylenediamines and aldehydes requires an oxidative reagent to generate the benzimidazole core. Various reagents such as nitrobenzene, benzoquinone, sodium metabisulfite, iodine/potassium iodide, $p$-toluenesulfonic acid and even air can be used for this purpose. Due to the availability of commercial aldehydes, the proposed method has been chosen as a general procedure for the synthesis. The versatile utility of substituted benzimidazole and the amide group, both being of pharmacological interest, and development of resistance to the existing anthelmintics prompted us to synthesize a series of benzimidazole derivatives containing the amide group in order to obtain a potential anthelmintic candidate.

\section{EXPERIMENTAL}

Melting points were determined in an open capillary tube and are uncorrected. The IR spectra were recorded as KBr discs using a JASCO FTIR 4100 spectrometer (Japan). ${ }^{1} \mathrm{H}$ NMR spectra were measured on a Varian-NMR-mercury 300 spectrometer (USA) using tetramethylsilane as internal standard and deuteriated dimethylsulphoxide as solvent and are expressed in parts per million $(\delta, \mathrm{ppm})$. Mass spectral data were recorded with an LC-MS spectrometer (Thermo Finnigan Model Discovery, Thermo Finnigan TSQ Quantum Discovery MAX Mass Spectrometer, USA). Microanalyses were performed using a Vario EL V300 elemental analyzer (Elemental Analysensysteme GmbH, Germany). The purity of the compounds was checked by TLC on pre-coated silica gel $\mathrm{G}\left(\mathrm{HF}_{254}\right)$ aluminium plates (Merck, USA) using benzene/ethyl acetate (4:1) and was visualized in a UV chamber.

\section{Syntheses}

2-(4-Substituted phenyl)-1H-benzimidazoles $(\mathbf{1} \boldsymbol{a}, \boldsymbol{b})$. General procedure. - Equimolar amounts of each $o$-phenylenediamine $(0.5 \mathrm{mmol})$ and the corresponding aromatic aldehyde $(0.5 \mathrm{mmol})$ were thoroughly mixed in $2 \mathrm{~mL}$ of dimethyl formamide. Sodium hydrogen sulfite $(0.15 \mathrm{mmol})$ was added and the mixture was stirred at $80{ }^{\circ} \mathrm{C}$. Completion of the reaction was monitored by TLC. The mixture was cooled to room temperature and added dropwise to $20 \mathrm{~mL}$ of water under vigorous stirring. The product, separated as a solid, was collected by filtration, washed with water, and dried.

Ethyl [2-(4-substituted phenyl)-1H-benzimidazol-1-yl] acetate $(2 \boldsymbol{a}, \boldsymbol{b})$. General procedure. A mixture of equimolar alkaline solution $(0.5 \mathrm{~mL}, 4 \mathrm{~mol} \mathrm{~L}-1 \mathrm{NaOH})$ of $1 \mathrm{H}$-benzimidazole $(0.01 \mathrm{~mol})$ in methanol $(50 \mathrm{~mL})$ and ethyl chloroacetate $(0.01 \mathrm{~mol})$ in methanol (30 $\mathrm{mL}$ ) was heated gently on a boiling water bath for $0.5 \mathrm{~h}$. The solid thus obtained on cooling was recrystallized from chloroform. 
R. Sawant and D. Kawade: Synthesis and biological evaluation of some novel 2-phenyl benzimidazole-1-acetamide derivatives as potential anthelmintic agents, Acta Pharm. 61 (2011) 353-361.

2-(4-Substituted phenyl)-1H-benzimidazole-1-yl acetamide derivatives (3a-r). General procedure. - To a solution of ethyl [2-(4-substituted phenyl)-1H-benzimidazol-1-yl] acetate $(0.01 \mathrm{~mol})$ dissolved in dry methanol $(50 \mathrm{~mL})$, substituted amines like $p$-amino benzoic acid, ethylamine, benzylamine, $p$-nitroaniline, $p$-aminophenol, $\alpha$-naphthalamine, aniline, $m$-nitroaniline and phenylhydrazine $(0.02 \mathrm{~mol})$ were added and the mixture was refluxed for $4-5 \mathrm{~h}$. The reaction mixture was cooled and the solid obtained was filtered, washed with a small quantity of cold methanol to give the final product.

\section{Anthelmintic activity}

The title compounds were tested for anthelmintic activity according to the method described by Dutta (14). Pheretima posthuma (earthworms authenticated by the SSGM College, Kopergaon, India) of approximately equal size $(6 \pm 1 \mathrm{~cm})$ were selected randomly for the present study. The worms were acclimatized to laboratory conditions before experimentation. The earthworms were divided into three groups of six earthworms each. Albendazole diluted with normal saline solution to obtain $20 \mathrm{mg} \mathrm{mL}^{-1}$ served as the standard and was poured into Petri dishes. The title compounds were dissolved in a minimum quantity of ethanol and diluted to prepare $20 \mathrm{mg} \mathrm{mL}^{-1}$ of each compound. Normal saline served as a control. The time taken for complete paralysis and death was recorded. The mean paralysis time and mean lethal time were calculated for each compound (each reading was taken in triplicate). The time taken for worms to become motionless was noted as paralysis time. To ascertain death, each worm was frequently subjected to external stimuli that stimulate and induce movement in earthworms, if alive.

\section{RESULTS AND DISCUSSION}

Some novel benzimidazole derivatives were synthesized in good yields using ambient reaction conditions and a simple work-up procedure. Physicochemical data of title compounds are shown in Table I. The structure of compounds was confirmed on the basis of IR, ${ }^{1} \mathrm{H}$ NMR, mass spectra and elemental analysis shown in Table II.

The purpose of the present study was to synthesize some novel 2-(4-substituted phenyl)-1H-benzimidazole-1-yl acetamide derivatives expected to have good anthelmintic activity (Scheme 1).

The starting materials, 2 -substituted- $1 H$-benzimidazoles (1a and $\mathbf{1 b}$ ), were prepared via oxidative condensation of $o$-phenylenediamine, the corresponding aromatic aldehyde and sodium metabisulfite. Structure of compounds $\mathbf{1 a}$ and $\mathbf{1 b}$ was confirmed by comparing their physical and spectral data with the reported ones. Nucleophilic substitution of compounds $\mathbf{1 a}$ and $\mathbf{1 b}$ yielded ethyl [2-(4-substituted phenyl)-1H-benzimidazol-1-yl] acetates ( $\mathbf{2} \mathbf{a}$ and $\mathbf{2 b}$ ). Treatment of compounds $\mathbf{2 a}$ and $\mathbf{2 b}$ with different substituted amines and phenylhydrazine resulted in the formation of 2-(4-substituted phenyl)- $1 \mathrm{H}$-benzimidazole-1-yl acetamide derivatives (3a-r). The IR spectra of title compounds (3a-r) showed a carbonyl stretching band between 1670-1780 $\mathrm{cm}^{-1}$ and in the NMR spectra the $\mathrm{CH}_{2}$ signal of acetamide moiety appeared at $\delta 4.96,5.03,4.92,4.90,4.96,4.96,4.96,4.96$, $4.96,4.96,5.03,4.92,4.90,4.96,4.96,4.96,4.96$ and 4.96 ppm, respectively. Mass spectra showed accurate $\mathrm{M}+1$ peaks for the title compounds (3a-r) and elemental analysis data 
R. Sawant and D. Kawade: Synthesis and biological evaluation of some novel 2-phenyl benzimidazole-1-acetamide derivatives as potential anthelmintic agents, Acta Pharm. 61 (2011) 353-361.

Table I. Physiochemical properties of the compounds $3 a-r$

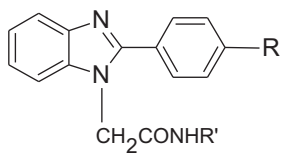

\begin{tabular}{|c|c|c|c|c|c|c|}
\hline Compd. & $\mathrm{R}$ & $\mathrm{R}^{\prime}$ & Mol. formula & $M_{\mathrm{r}}$ & Yield (\%) & M.p. $\left({ }^{\circ} \mathrm{C}\right)$ \\
\hline $3 a$ & $\mathrm{NO}_{2}$ & $-\mathrm{Ph}(p) \mathrm{COOH}$ & $\mathrm{C}_{22} \mathrm{H}_{16} \mathrm{~N}_{4} \mathrm{O}_{5}$ & 416.38 & 53.12 & 305 \\
\hline $3 b$ & $\mathrm{NO}_{2}$ & -NHPh & $\mathrm{C}_{21} \mathrm{H}_{17} \mathrm{~N}_{5} \mathrm{O}_{3}$ & 387.39 & 64.6 & 284 \\
\hline $3 c$ & $\mathrm{NO}_{2}$ & Et & $\mathrm{C}_{17} \mathrm{H}_{16} \mathrm{~N}_{4} \mathrm{O}_{3}$ & 324.33 & 80.55 & 295 \\
\hline $3 d$ & $\mathrm{NO}_{2}$ & $-\mathrm{CH}_{2} \mathrm{Ph}$ & $\mathrm{C}_{22} \mathrm{H}_{18} \mathrm{~N}_{4} \mathrm{O}_{3}$ & 386.40 & 57.14 & 305 \\
\hline $3 e$ & $\mathrm{NO}_{2}$ & $-\mathrm{Ph}(p) \mathrm{NO}_{2}$ & $\mathrm{C}_{21} \mathrm{H}_{15} \mathrm{~N}_{5} \mathrm{O}_{5}$ & 417.37 & 58.33 & 285 \\
\hline $3 f$ & $\mathrm{NO}_{2}$ & $-\mathrm{Ph}(p) \mathrm{OH}$ & $\mathrm{C}_{21} \mathrm{H}_{16} \mathrm{~N}_{4} \mathrm{O}_{4}$ & 388.37 & 54.62 & 290 \\
\hline $3 g$ & $\mathrm{NO}_{2}$ & naphthyl & $\mathrm{C}_{25} \mathrm{H}_{18} \mathrm{~N}_{4} \mathrm{O}_{3}$ & 422.43 & 58.24 & 285 \\
\hline $3 \mathrm{~h}$ & $\mathrm{NO}_{2}$ & $\mathrm{Ph}$ & $\mathrm{C}_{21} \mathrm{H}_{16} \mathrm{~N}_{4} \mathrm{O}_{3}$ & 372.37 & 65.49 & 288 \\
\hline $3 \mathbf{i}$ & $\mathrm{NO}_{2}$ & $-\mathrm{Ph}(m) \mathrm{NO}_{2}$ & $\mathrm{C}_{21} \mathrm{H}_{15} \mathrm{~N}_{5} \mathrm{O}_{5}$ & 417.37 & 62.50 & 310 \\
\hline $3 \mathbf{j}$ & $\mathrm{Cl}$ & $-\mathrm{Ph}(p) \mathrm{COOH}$ & $\mathrm{C}_{22} \mathrm{H}_{16} \mathrm{ClN}_{3} \mathrm{O}_{3}$ & 405.83 & 54.68 & 285 \\
\hline $3 k$ & $\mathrm{Cl}$ & -NHPh & $\mathrm{C}_{21} \mathrm{H}_{17} \mathrm{ClN}_{4} \mathrm{O}$ & 376.83 & 66.47 & 290 \\
\hline 31 & $\mathrm{Cl}$ & Et & $\mathrm{C}_{17} \mathrm{H}_{16} \mathrm{ClN}_{3} \mathrm{O}$ & 313.78 & 79.28 & 300 \\
\hline $3 m$ & $\mathrm{Cl}$ & $-\mathrm{CH}_{2} \mathrm{Ph}$ & $\mathrm{C}_{22} \mathrm{H}_{18} \mathrm{CLN}_{3} \mathrm{O}$ & 375.85 & 68.53 & 285 \\
\hline $3 n$ & $\mathrm{Cl}$ & $-\mathrm{Ph}(p) \mathrm{NO}_{2}$ & $\mathrm{C}_{21} \mathrm{H}_{15} \mathrm{ClN}_{4} \mathrm{O}_{3}$ & 406.82 & 58.14 & 260 \\
\hline 30 & $\mathrm{Cl}$ & $-\mathrm{Ph}(p) \mathrm{OH}$ & $\mathrm{C}_{21} \mathrm{H}_{16} \mathrm{ClN}_{3} \mathrm{O}_{2}$ & 377.82 & 77.91 & 290 \\
\hline $3 p$ & $\mathrm{Cl}$ & naphthyl & $\mathrm{C}_{25} \mathrm{H}_{18} \mathrm{ClN}_{3} \mathrm{O}$ & 411.88 & 70.76 & 284 \\
\hline $3 q$ & $\mathrm{Cl}$ & $\mathrm{Ph}$ & $\mathrm{C}_{21} \mathrm{H}_{16} \mathrm{ClN}_{3} \mathrm{O}_{2}$ & 361.82 & 84.54 & 288 \\
\hline $3 r$ & $\mathrm{Cl}$ & $-\mathrm{Ph}(m) \mathrm{NO}_{2}$ & $\mathrm{C}_{21} \mathrm{H}_{15} \mathrm{ClN}_{4} \mathrm{O}_{3}$ & 406.82 & 77.66 & 297 \\
\hline
\end{tabular}
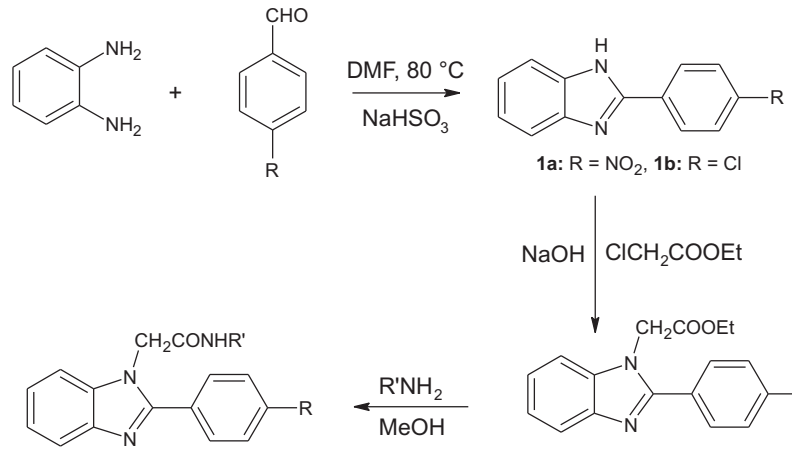

$$
\mathrm{R} \text { and } \mathrm{R}^{\prime} \text { as in Table I. }
$$

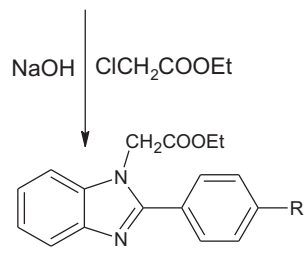

2a: $\mathrm{R}=\mathrm{NO}_{2}, \mathbf{2 b}: \mathrm{R}=\mathrm{Cl}$

Scheme 1 
R. Sawant and D. Kawade: Synthesis and biological evaluation of some novel 2-phenyl benzimidazole-1-acetamide derivatives as potential anthelmintic agents, Acta Pharm. 61 (2011) 353-361.

Table II. Spectral data of title compounds

\begin{tabular}{|c|c|c|c|c|c|c|}
\hline \multirow{2}{*}{ Compd. } & \multirow{2}{*}{$\begin{array}{c}\text { FTIR } \\
\left(\mathrm{KBr}, \mathrm{cm}^{-1}\right)\end{array}$} & \multirow{2}{*}{$\begin{array}{c}{ }^{1} \mathrm{H} \text { NMR } \\
\left(\mathrm{DMSO}-d_{6}, \delta, \mathrm{ppm}\right)\end{array}$} & \multirow{2}{*}{$\begin{array}{l}\mathrm{M}+1 \\
\text { peak }\end{array}$} & \multicolumn{3}{|c|}{$\begin{array}{l}\text { Elemental analysis } \\
\text { Calcd./found (\%) }\end{array}$} \\
\hline & & & & C & $\mathrm{H}$ & $\mathrm{N}$ \\
\hline $3 a$ & $\begin{array}{l}3362.4(\mathrm{OH}), 1719.2 \\
(\mathrm{C}=\mathrm{O}), 16011.2(\mathrm{C}=\mathrm{N}), \\
1515.8\left(\mathrm{Asy} A r-\mathrm{NO}_{2}\right), \\
1335.5\left(\mathrm{Sym} \mathrm{Ar}-\mathrm{NO}_{2}\right) \\
1282.4(\mathrm{C}-\mathrm{O}), 1104.1 \\
(\mathrm{C}-\mathrm{N})\end{array}$ & $\begin{array}{l}4.96\left(\mathrm{~d}, 2 \mathrm{H}, \mathrm{CH}_{2}\right) \\
7.22-8.09(\mathrm{~m}, 12 \mathrm{H} \\
\mathrm{Ar}), 9.24(\mathrm{t}, 1 \mathrm{H}, \mathrm{NH}) \\
9.93(\mathrm{~s}, 1 \mathrm{H}, \mathrm{OH})\end{array}$ & 417 & $\begin{array}{l}63.46 \\
63.40\end{array}$ & $\begin{array}{l}3.87 \\
3.81\end{array}$ & $\begin{array}{l}13.46 \\
13.46\end{array}$ \\
\hline $3 b$ & $\begin{array}{l}3044.1(\mathrm{Ar}-\mathrm{CH}), 1773.2 \\
(\mathrm{C}=\mathrm{O}), 1605.5(\mathrm{C}=\mathrm{N}), \\
1515.8\left(\text { Asy Ar- } \mathrm{NO}_{2}\right) \\
1437.7(\mathrm{C}-\mathrm{N}), 1341.3 \\
\left(\text { Sym Ar- } \mathrm{NO}_{2}\right), 1107.9 \\
(\mathrm{C}-\mathrm{N})\end{array}$ & $\begin{array}{l}5.03\left(\mathrm{~d}, 2 \mathrm{H}, \mathrm{CH}_{2}\right) \\
7.22-8.09(\mathrm{~m}, 13 \mathrm{H} \\
\mathrm{Ar}), 9.28(\mathrm{t}, 1 \mathrm{H}, \mathrm{NH}) \\
7.549(\mathrm{~s}, 1 \mathrm{H}, \mathrm{NH})\end{array}$ & 388 & $\begin{array}{l}65.11 \\
65.01\end{array}$ & $\begin{array}{l}4.42 \\
4.39\end{array}$ & $\begin{array}{l}18.08 \\
18.18\end{array}$ \\
\hline $3 c$ & $\begin{array}{l}3038.3(\mathrm{Ar}-\mathrm{CH}), 2948.6 \\
\left(\mathrm{CH}_{2} \text { and } \mathrm{CH}\right), 1605.5 \\
(\mathrm{C}=\mathrm{N}), 1509.0(\mathrm{Asy} \\
\left.\mathrm{Ar}-\mathrm{NO}_{2}\right), 1437.7(\mathrm{C}-\mathrm{N}) \\
1335.5\left(\mathrm{Sym} \text { Ar- } \mathrm{NO}_{2}\right)\end{array}$ & $\begin{array}{l}1.25\left(\mathrm{~m}, 3 \mathrm{H}, \mathrm{CH}_{3}\right), \\
3.63\left(\mathrm{~m}, 2 \mathrm{H}, \mathrm{CH}_{2}\right), \\
4.92\left(\mathrm{~d}, 2 \mathrm{H}, \mathrm{CH}_{2}\right), \\
4.09(\mathrm{~m}, 1 \mathrm{H}, \mathrm{NH}) \\
7.19-8.09(\mathrm{~m}, 8 \mathrm{H}, \mathrm{Ar})\end{array}$ & 325 & $\begin{array}{l}62.95 \\
62.90\end{array}$ & $\begin{array}{l}4.97 \\
4.97\end{array}$ & $\begin{array}{l}17.27 \\
17.23\end{array}$ \\
\hline $3 d$ & 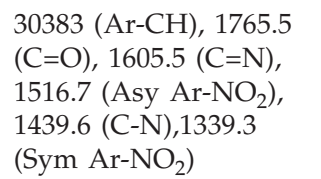 & $\begin{array}{l}4.22\left(\mathrm{~m}, 2 \mathrm{H}, \mathrm{CH}_{2}\right), \\
4.90\left(\mathrm{~d}, 2 \mathrm{H}, \mathrm{CH}_{2}\right), \\
7.15-8.09(\mathrm{~m}, 13 \mathrm{H}, \\
\text { Ar), } 7.24(\mathrm{~s}, 1 \mathrm{H}, \mathrm{NH})\end{array}$ & 388 & $\begin{array}{l}68.38 \\
68.31\end{array}$ & $\begin{array}{l}4.70 \\
4.67\end{array}$ & $\begin{array}{l}14.50 \\
14.43\end{array}$ \\
\hline $3 e$ & $\begin{array}{l}3032.5(\text { Ar-CH }), 1605.5 \\
(\mathrm{C}=\mathrm{N}), 1509.0(\text { Asy } \\
\left.\text { Ar-NO }_{2}\right), 1431.9(\mathrm{C}-\mathrm{N}), \\
1341.3\left(\text { Sym Ar- } \mathrm{NO}_{2}\right)\end{array}$ & $\begin{array}{l}4.96\left(\mathrm{~d}, 2 \mathrm{H}, \mathrm{CH}_{2}\right) \\
7.22-8.09(\mathrm{~m}, 12 \mathrm{H} \\
\mathrm{Ar}), 9.237(\mathrm{t}, 1 \mathrm{H}, \mathrm{NH})\end{array}$ & 418 & $\begin{array}{l}60.43 \\
60.40\end{array}$ & $\begin{array}{l}3.62 \\
3.68\end{array}$ & $\begin{array}{l}16.78 \\
16.71\end{array}$ \\
\hline $3 f$ & $\begin{array}{l}3362.4(\mathrm{OH}), 1719.2 \\
(\mathrm{C}=\mathrm{O}), 1605.5(\mathrm{C}=\mathrm{N}) \\
1509.0\left(\text { Asy } \mathrm{Ar}-\mathrm{NO}_{2}\right) \\
1431.9(\mathrm{C}-\mathrm{N}), 1281.5 \\
(\mathrm{C}-\mathrm{O}), 1107.9(\mathrm{C}-\mathrm{N})\end{array}$ & $\begin{array}{l}4.96\left(\mathrm{~d}, 2 \mathrm{H}, \mathrm{CH}_{2}\right), \\
5.11(\mathrm{~s}, 1 \mathrm{H}, \mathrm{OH}) \\
7.22-8.09(\mathrm{~m}, 12 \mathrm{H}, \\
\mathrm{Ar}), 9.24(\mathrm{t}, 1 \mathrm{H}, \mathrm{NH})\end{array}$ & 390 & $\begin{array}{l}64.46 \\
64.46\end{array}$ & $\begin{array}{l}4.15 \\
4.11\end{array}$ & $\begin{array}{l}14.43 \\
14.35\end{array}$ \\
\hline $3 g$ & $\begin{array}{l}1683.6(\mathrm{C}=0), 1611.2 \\
(\mathrm{C}=\mathrm{N}), 1515.8(\text { Asy } \\
\left.\mathrm{Ar}^{-N_{2}}\right), 1437.7(\mathrm{C}-\mathrm{N}), \\
1341.3\left(\text { Sym Ar- } \mathrm{NO}_{2}\right)\end{array}$ & $\begin{array}{l}4.96\left(\mathrm{~d}, 2 \mathrm{H}, \mathrm{CH}_{2}\right), \\
7.22-8.09(\mathrm{~m}, 15 \mathrm{H} \\
\text { Ar), } 9.62(\mathrm{t}, 1 \mathrm{H}, \mathrm{NH})\end{array}$ & 423 & $\begin{array}{l}71.08 \\
71.10\end{array}$ & $\begin{array}{l}4.29 \\
4.27\end{array}$ & $\begin{array}{l}13.26 \\
13.26\end{array}$ \\
\hline $3 h$ & 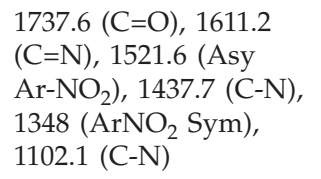 & $\begin{array}{l}4.96\left(\mathrm{~d}, 2 \mathrm{H}, \mathrm{CH}_{2}\right) \\
7.15-8.09(\mathrm{~m}, 13 \mathrm{H} \\
\mathrm{Ar}), 9.24(\mathrm{t}, 1 \mathrm{H}, \mathrm{NH})\end{array}$ & 373 & $\begin{array}{l}67.73 \\
67.78\end{array}$ & $\begin{array}{l}4.33 \\
4.23\end{array}$ & $\begin{array}{l}15.05 \\
15.03\end{array}$ \\
\hline $3 \mathbf{i}$ & 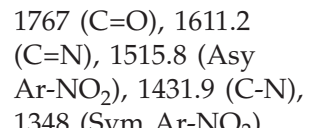 & $\begin{array}{l}4.96\left(\mathrm{~d}, 2 \mathrm{H}, \mathrm{CH}_{2}\right), \\
7.22-8.09(\mathrm{~m}, 12 \mathrm{H}, \\
\text { Ar), } 9.37(\mathrm{t}, 1 \mathrm{H}, \mathrm{NH})\end{array}$ & 418 & $\begin{array}{l}60.43 \\
60.41\end{array}$ & $\begin{array}{l}3.62 \\
3.66\end{array}$ & $\begin{array}{l}16.78 \\
16.77\end{array}$ \\
\hline
\end{tabular}


R. Sawant and D. Kawade: Synthesis and biological evaluation of some novel 2-phenyl benzimidazole-1-acetamide derivatives as potential anthelmintic agents, Acta Pharm. 61 (2011) 353-361.

\begin{tabular}{|c|c|c|c|c|c|c|}
\hline $3 \mathbf{j}$ & $\begin{array}{l}1719.2(\mathrm{C}=\mathrm{O}), 1425.1 \\
(\mathrm{C}-\mathrm{N}), 1317.1(\mathrm{C}-\mathrm{O}), \\
754.0(\mathrm{C}-\mathrm{Cl})\end{array}$ & $\begin{array}{l}4.96\left(\mathrm{~d}, 2 \mathrm{H}, \mathrm{CH}_{2}\right), \\
9.24(\mathrm{t}, 1 \mathrm{H}, \mathrm{NH}), \\
7.22-7.83(\mathrm{~m}, 12 \mathrm{H}, \\
\text { Ar), } 9.93(\mathrm{~s}, 1 \mathrm{H}, \mathrm{OH})\end{array}$ & 407 & $\begin{array}{l}65.11 \\
65.09\end{array}$ & $\begin{array}{l}3.97 \\
3.94\end{array}$ & $\begin{array}{l}10.35 \\
10.35\end{array}$ \\
\hline $3 k$ & $\begin{array}{l}3055.7(\mathrm{Ar}-\mathrm{CH}), 1605.5 \\
(\mathrm{C}=\mathrm{N}), 1455.0(\mathrm{C}-\mathrm{N}) \\
735.7(\mathrm{C}-\mathrm{Cl})\end{array}$ & $\begin{array}{l}5.03\left(\mathrm{~d}, 2 \mathrm{H}, \mathrm{CH}_{2}\right) \\
7.05-7.79(\mathrm{~m}, 13 \mathrm{H}, \\
\mathrm{Ar}), 7.55(\mathrm{~s}, 1 \mathrm{H}, \mathrm{NH}) \\
11.28(\mathrm{t}, 1 \mathrm{H}, \mathrm{NH})\end{array}$ & 378 & $\begin{array}{l}66.93 \\
66.94\end{array}$ & $\begin{array}{l}4.55 \\
4.51\end{array}$ & $\begin{array}{l}14.87 \\
14.85\end{array}$ \\
\hline 31 & $\begin{array}{l}3049.9(\mathrm{Ar}-\mathrm{CH}), 2913.9 \\
\left(\mathrm{CH}_{2}\right), 1605.5(\mathrm{C}=\mathrm{N}) \\
1268.0(\mathrm{C}-\mathrm{N}), 747.3 \\
(\mathrm{C}-\mathrm{Cl})\end{array}$ & $\begin{array}{l}1.25\left(\mathrm{~m}, 3 \mathrm{H}, \mathrm{CH}_{3}\right) \\
3.63\left(\mathrm{~m}, 2 \mathrm{H}, \mathrm{CH}_{2}\right) \\
4.19(\mathrm{~m}, 1 \mathrm{H}, \mathrm{NH}) \\
4.92\left(\mathrm{~d}, 2 \mathrm{H}, \mathrm{CH}_{2}\right) \\
7.20-7.79(\mathrm{~m}, 8 \mathrm{H}, \\
\text { ArH) }\end{array}$ & 315 & $\begin{array}{l}65.07 \\
65.02\end{array}$ & $\begin{array}{l}5.14 \\
5.11\end{array}$ & $\begin{array}{l}13.39 \\
13.36\end{array}$ \\
\hline $3 m$ & $\begin{array}{l}3055.7(\mathrm{Ar}-\mathrm{CH}), 1771.3 \\
(\mathrm{C}=\mathrm{O}), 1428.0(\mathrm{C}-\mathrm{N}) \\
741.5(\mathrm{C}-\mathrm{Cl})\end{array}$ & $\begin{array}{l}4.22\left(\mathrm{~m}, 2 \mathrm{H}, \mathrm{CH}_{2}\right), \\
4.90\left(\mathrm{~d}, 2 \mathrm{H}, \mathrm{CH}_{2}\right), \\
7.15-7.79(\mathrm{~m}, 13 \mathrm{H}, \\
\mathrm{Ar}), 7.239(\mathrm{~s}, 1 \mathrm{H}, \\
\mathrm{NH})\end{array}$ & 377 & $\begin{array}{l}68.38 \\
68.34\end{array}$ & $\begin{array}{l}4.70 \\
4.75\end{array}$ & $\begin{array}{l}14.50 \\
14.46\end{array}$ \\
\hline $3 n$ & $\begin{array}{l}3062.4(\mathrm{Ar}-\mathrm{CH}), 1771.3 \\
(\mathrm{C}=\mathrm{O}), 1588.1(\mathrm{C}=\mathrm{N}) \\
1428.0(\mathrm{C}-\mathrm{N}), 1322.0 \\
\left(\mathrm{Sym} \mathrm{NO}_{2}\right), 741.5(\mathrm{C}-\mathrm{Cl})\end{array}$ & $\begin{array}{l}4.96\left(\mathrm{~d}, 2 \mathrm{H}, \mathrm{CH}_{2}\right), \\
7.22-8.09(\mathrm{~m}, 12 \mathrm{H}, \\
\mathrm{Ar}), 9.24(\mathrm{t}, 1 \mathrm{H}, \mathrm{NH})\end{array}$ & 408 & $\begin{array}{l}62.00 \\
62.05\end{array}$ & $\begin{array}{l}3.72 \\
3.72\end{array}$ & $\begin{array}{l}13.77 \\
13.75\end{array}$ \\
\hline 30 & $\begin{array}{l}3345.9(\mathrm{Ar}-\mathrm{CH}), 3281.3 \\
(-\mathrm{OH}), 1759.73(\mathrm{C}=\mathrm{O}) \\
1605.5(\mathrm{C}=\mathrm{N}), 1428.0 \\
(\mathrm{C}-\mathrm{N}), 1243.8(\mathrm{C}-\mathrm{O}) \\
741.5(\mathrm{C}-\mathrm{Cl})\end{array}$ & $\begin{array}{l}4.96\left(\mathrm{~d}, 2 \mathrm{H}, \mathrm{CH}_{2}\right), \\
5.12(\mathrm{~s}, 1 \mathrm{H}, \mathrm{OH}) \\
7.22-7.79(\mathrm{~m}, 12 \mathrm{H}, \\
\text { Ar), } 9.24(\mathrm{t}, 1 \mathrm{H}, \mathrm{NH})\end{array}$ & 379 & $\begin{array}{l}66.76 \\
66.74\end{array}$ & $\begin{array}{l}4.27 \\
4.21\end{array}$ & $\begin{array}{l}11.12 \\
11.18\end{array}$ \\
\hline $3 p$ & $\begin{array}{l}3055.7(\mathrm{Ar}-\mathrm{CH}), 1671.0 \\
(\mathrm{C}=\mathrm{O}), 1605.5(\mathrm{C}=\mathrm{N}) \\
1422.2(\mathrm{C}-\mathrm{N}), 1273.8 \\
(\mathrm{C}-\mathrm{N}), 747.2(\mathrm{C}-\mathrm{Cl})\end{array}$ & $\begin{array}{l}4.96\left(\mathrm{~d}, 2 \mathrm{H}, \mathrm{CH}_{2}\right), \\
7.22-8.09(\mathrm{~m}, 15 \mathrm{H}, \\
\mathrm{Ar}), 9.62(\mathrm{t}, 1 \mathrm{H}, \mathrm{NH})\end{array}$ & 413 & $\begin{array}{l}72.90 \\
72.89\end{array}$ & $\begin{array}{l}4.40 \\
4.35\end{array}$ & $\begin{array}{l}10.20 \\
10.26\end{array}$ \\
\hline $3 q$ & $\begin{array}{l}3055.7(\mathrm{Ar}-\mathrm{CH}), 1771.3 \\
(\mathrm{C}=\mathrm{O}), 1428.0(\mathrm{C}-\mathrm{N}) \\
747.3(\mathrm{C}-\mathrm{Cl})\end{array}$ & $\begin{array}{l}4.96\left(\mathrm{~d}, 2 \mathrm{H}, \mathrm{CH}_{2}\right) \\
7.15-7.79(\mathrm{~m}, 13 \mathrm{H} \\
\mathrm{Ar}), 9.237(\mathrm{t}, 1 \mathrm{H}, \mathrm{NH})\end{array}$ & 363 & $\begin{array}{l}69.71 \\
69.70\end{array}$ & $\begin{array}{l}4.46 \\
4.42\end{array}$ & $\begin{array}{l}11.61 \\
11.61\end{array}$ \\
\hline $3 r$ & $\begin{array}{l}3049.9(\mathrm{Ar}-\mathrm{CH}), 1759.7 \\
(\mathrm{C}=\mathrm{O}), 1599.7(\mathrm{C}=\mathrm{N}) \\
1428.0(\mathrm{C}-\mathrm{N}), 1322.0 \\
\left(\mathrm{Sym} \mathrm{NO}_{2}\right), 747.3(\mathrm{C}-\mathrm{Cl})\end{array}$ & $\begin{array}{l}4.96\left(\mathrm{~d}, 2 \mathrm{H}, \mathrm{CH}_{2}\right) \\
7.22-8.79(\mathrm{~m}, 12 \mathrm{H} \\
\mathrm{Ar}), 9.27(\mathrm{t}, 1 \mathrm{H}, \mathrm{NH})\end{array}$ & 408 & $\begin{array}{l}62.00 \\
62.05\end{array}$ & $\begin{array}{l}3.72 \\
3.70\end{array}$ & $\begin{array}{l}13.77 \\
13.72\end{array}$ \\
\hline
\end{tabular}

showed a less than $0.99 \%$ difference between the calculated and found results. All these facts confirm the structure of the title compounds.

To evaluate the anthelmintic activity, adult Indian earthworms (Pheretima posthuma) were used for the experimental protocol due to their anatomical and physiological resemblance to the intestinal roundworm parasites in humans. Observations were made for the time taken to paralysis and death of individual worms (Table III). Paralysis occurred when the worms did not revive even in normal saline. Death was declared when the worms lost motility, followed by fading away of their body colors. Compounds 3a, 
R. Sawant and D. Kawade: Synthesis and biological evaluation of some novel 2-phenyl benzimidazole-1-acetamide derivatives as potential anthelmintic agents, Acta Pharm. 61 (2011) 353-361.

$3 c, 3 d, 3 f, 3 h, 3 k, 3 n$ and $3 q$ were found more active to paralyze worms than $3 b, 3 g, 3 p$ and $3 \mathbf{r}$ whereas compounds $3 \mathbf{c}, 3 \mathbf{e}, 3 \mathbf{j}, 3 \mathbf{l}$ and $\mathbf{3 q}$ were more potent to cause death of worms than albendazole. Compound $3 \mathrm{c}$ was the most potent to paralyze worms as well as to cause death of worms, which revealed that aliphatic acetamides with the presence of the electron withdrawing polar group at the fourth position of 2-phenyl ring of benzimidazole-1-acetamide derivatives resulted in higher anthelmintic activity.

Table III. Anthelmintic activity of compounds $3 a-r^{a}$

\begin{tabular}{ccc}
\hline Compd. $^{\text {b }}$ & ${\text { Paralysis time }(\mathrm{min})^{\mathrm{c}}}$ & Death time $(\mathrm{min})^{\mathrm{c}}$ \\
\hline Albendazole & $21.43 \pm 1.16$ & $55.44 \pm 1.65$ \\
3a & $19.29 \pm 2.89$ & $58.25 \pm 2.38$ \\
3b & $31.37 \pm 1.10$ & $61.51 \pm 1.64$ \\
3c & $14.60 \pm 3.53$ & $47.22 \pm 1.03$ \\
3d & $17.27 \pm 2.81$ & $58.43 \pm 1.15$ \\
3e & $25.31 \pm 3.49$ & $55.12 \pm 5.12$ \\
3f & $20.39 \pm 1.19$ & $55.52 \pm 2.7$ \\
3g & $29.16 \pm 1.12$ & $86.79 \pm 2.95$ \\
3h & $17.23 \pm 2.35$ & $63.72 \pm 1.73$ \\
3i & $28.29 \pm 1.73$ & $72.36 \pm 3.44$ \\
3j & $22.53 \pm 2.17$ & $53.48 \pm 1.62$ \\
3k & $17.47 \pm 1.08$ & $58.43 \pm 1.15$ \\
31 & $26.76 \pm 3.40$ & $51.51 \pm 1.76$ \\
3m & $25.36 \pm 2.95$ & $63.38 \pm 1.72$ \\
3n & $19.46 \pm 3.03$ & $58.26 \pm 3.55$ \\
3o & $23.58 \pm 4.01$ & $58.71 \pm 2.33$ \\
3p & $30.40 \pm 1.77$ & $69.22 \pm 1.13$ \\
3q & $20.36 \pm 1.59$ & $51.38 \pm 1.09$ \\
3r & $34.44 \pm 2.32$ & $59.45 \pm 1.15$ \\
\hline
\end{tabular}

${ }^{\text {a }}$ Control worms were alive up to 24 hours of the experiment.

${ }^{\mathrm{b}}$ Concentration in normal saline: $20 \mu \mathrm{g} \mathrm{mL} \mathrm{m}^{-1}$.

c Values are expressed as mean \pm SEM, $n=6$.

\section{CONCLUSIONS}

The present study has demonstrated that 2-phenyl benzimidazole-1-acetamide derivatives are potential novel anthelmintic agents. Further work is in progress to trace the mechanisms of action and structural requirements of this series of compounds by computational methods. 
R. Sawant and D. Kawade: Synthesis and biological evaluation of some novel 2-phenyl benzimidazole-1-acetamide derivatives as potential anthelmintic agents, Acta Pharm. 61 (2011) 353-361.

\section{REFERENCES}

1. C. Kus, G. A. Kilcigil, B. C. Eke and M. I. Can, Synthesis and antioxidant properties of some novel benzimidazole derivatives on lipid peroxidation in the rat liver, Arch. Pharm. Res. 27 (2004) 156-163; DOI: 10.1007/BF02980099.

2. C. Kus, G. A. Kilcigil, S. Ozbey and F. B. Kaynak, Synthesis and antioxidant properties of novel $\mathrm{N}$-methyl-1,3,4-thiadiazol-2-amine and 4-methyl-2H-1,2,4-triazole-3(4H)-thione derivatives of benzimidazole class, Bioorg. Med. Chem. Lett. 16 (2008) 4294-4303; DOI: 10.1016/j.bmc.2008.02.077.

3. F. P. Alonso, H. J. Cook, J. P. Villanueva, J. C. Piliado, S. R. Morales, G. P. Hernandez, N. L. Balbiaux, A. H. Campos, R. Castillo and F. H. Luis, Synthesis and in vitro cysticidal activity of new benzimidazole derivatives, Eur. J. Med. Chem. 44 (2009) 1794-1800; DOI: 10.1016/j.ejmech. 2008.05.005.

4. D. P. Jasmer, C. Yao, A. Rehman and S. Johnson, Multiple lethal effects induced by a benzimidazole anthelmintic in the anterior intestine of the nematode Haemonchus contortus, Mol. Biochem. Parasit. 105 (2000) 81-90; DOI: 10.1016/S0166-6851(99)00169-3.

5. H. Xiangming, M. Huiqiang and W. Yulu, $p$-TsOH catalyzed synthesis of 2-arylsubstituted benzimidazoles, ARKIVOC 13 (2007) 150-154.

6. K. F. Ansari and C. Lal, Synthesis and evaluation of some new benzimidazole derivatives as potential antimicrobial agents, Eur. J. Med. Chem. 44 (2009) 2294-2299; DOI: 10.1016/j.ejmech.2008. 01.022 .

7. M. L. Richards, S. C. Lio, A. Sinha, H. Banie, R. J. Thomas, M. Major, M. Tanji and J. C. Sircar, Substituted 2-phenyl-benzimidazole derivatives: novel compounds that suppress key markers of allergy, Eur. J. Med. Chem. 41 (2006) 950-969; DOI: 10.1016/j.ejmech.2006.03.014.

8. I. Kerimov, G. L. Kilcigil, B. Caneke, N. Altanlar and M. M. Scan, Synthesis, antifungal and antioxidant screening of some novel benzimidazole derivatives, J. Enz. Inhib. Med. Chem. 22 (2007) 696-701; DOI: 10.1080/14756360701228558.

9. J. R. Kumar, J. Jawahar and D. P. Pathak, Synthesis of benzimidazole derivatives as anti-hypertensive agents, E-J. Chem. 3 (2006) 278-285.

10. M. Hranjec, K. Starcevic, I. Piantanida, M. Kralj, M. Marjanovic, M. Hasani, G. Westman and G. K. Zamola, Synthesis, antitumor evaluation and DNA binding studies of novel amidino-benzimidazolyl substituted derivatives of furyl-phenyl- and thienyl-phenyl-acrylates, naphthofurans and naphthothiophenes, Eur. J. Med. Chem. 43 (2008) 2877-2890; DOI: 10.1016/j.ejmech.2008.02.010.

11. S. S. Mahajan and R. G. Nandre, The synthesis of 2-mercapto-5-methoxybenzimidazole, Indian J. Chem. 45B (2006) 1756-1758; DOI: 10.1002/chin.200646161.

12. O. Algul, A. Kaessler, Y. Apcin, A. Yilmaz and J. Jose, Comparative studies on conventional and microwave synthesis of some benzimidazole, benzothiazole and indole derivatives and testing on inhibition of hyaluronidase, Molecules 13 (2008) 736-748; DOI: 10.3390/molecules13040736.

13. H. J. Hall and D. R. Kamm, Synthesis of benzimidazoles from anils of $o$-azidoaniline, J. Org. Chem. 30 (1965) 2092-2093; DOI: 10.1021/jo01017a533.

14. S. Dutta, Synthesis and anthelmintic activity of some novel 2-substituted-4,5-diphenyl imidazoles, Acta Pharm. 60 (2010) 229-235; DOI: 10.2478/v10007-010-0011-1. 
R. Sawant and D. Kawade: Synthesis and biological evaluation of some novel 2-phenyl benzimidazole-1-acetamide derivatives as potential anthelmintic agents, Acta Pharm. 61 (2011) 353-361.

\section{$S A \check{Z} E T A K$}

\section{Sinteza i biološko vrednovanje novih derivata 2-fenil-benzimidazol-1-acetamida kao potencijalnih anthelmintika}

RAMESH SAWANT i DEEPALI KAWADE

U radu je opisana sinteza derivata 2-fenil-benzimidazol-1-acetamida i ispitivanje njihovog anthelmintičkog djelovanja na odrasle indijske gliste, Pheretima posthuma. Struktura sintetiziranih spojeva određena je elementarnom analizom i spektroskopskim metodama. Spojevi 4-(\{[2-(4-nitrofenil)-1H-benzimidazol-1-il]acetil\}amino) benzojeva kiselina (3a), $N$-etil-2-[2-(4-nitrofenil)-1H-benzimidazol-1-il] acetamid (3c), $N$-benzil-2-[2-(4-nitrofenil)-1H-benzimidazol-1-il] acetamid (3d), $N$-(4-hidroksifenil)-2-[2-(4-nitrofenil)- $1 H$-benzimidazol-1-il] acetamid (3f), 2-[2-(4-nitrofenil)-1H-benzimidazol-1-il]- $N$-fenilacetamid (3h), 2-[2-(4-klorfenil)-1H-benzimidazol-1-il]-N'-fenilacetohidrazid (3k), 2-[2-(4-klorfenil)-1H-benzimidazol-1-il]-N-(4-nitrofenil) acetamid (3n) i 2-[2-(4-klorfenil)-1H-benzimidazol-1-il]- $N$-fenilacetamid (3q) jače paraliziraju gliste, a $N$-etil-2-[2-(4-nitrofenil)-1H-benzimidazol-1-il] acetamid (3c), 2-[2-(4-nitrofenil)-1H-benzimidazol-1-il]- $N$-fenilacetamid (3h), 4-(\{[2-(4-klorfenil)-1H-benzimidazol-1-il]acetil\}amino) benzojeva kiselina (3j), 2-[2-(4-klorfenil)-1H-benzimidazol-1-il]- $N$-etilacetamid (3l) i 2-[2-(4-klorfenil)-1H-benzimidazol-1-il]$\mathrm{N}$-fenilacetamid (3q) učinkovitije usmrćuju gliste nego anthelmintik albendazol.

Ključne riječi: benzimidazol, anthelmintičko djelovanje, Pheretima posthuma

Department of Pharmaceutical Chemistry and PG Studies, PDVVPF's College of Pharmacy, Post-MIDC

Vilad Ghat, Ahmednagar-414111, India 